\title{
New Splitting Iterative Method for Fuzzy Linear Systems
}

\section{Shiheng Wang* and Deqi Lian}

\author{
Nanyang Vocational College of Agriculture, Nanyang 473000, P.R. China \\ the corresponding author, email: wsh1965168@qq.com
}

Keywords: Splitting iterative method; Fuzzy linear system; Jacobi method.

Abstract. In this paper, a new splitting iterative method is discussed for solving fuzzy linear systems of equations, where the coefficient matrix is crisp and the right-hand side is fuzzy vector. The convergence theorem is provided with numerical examples to illustrate the method.

\section{Introduction}

Fuzzy linear systems (FLSs) play an important role in many fields, such as control problems, information, physics, statistics, engineering, economics, finance and even social sciences. Thus, many authors establish mathematical models and numerical methods for solving FLSs [4].

Friedman et al. [7] proposed a general model for solving a class of $n \times n$ FLSs

$$
A x=y \text { or }\left\{\begin{array}{l}
a_{11} x_{1}+a_{12} x_{2}++a_{1 n} x_{n}=y_{1}, \\
a_{21} x_{1}+a_{22} x_{2}+\quad+a_{2 n} x_{n}=y_{2}, \\
a_{n 1} x_{1}+a_{n 2} x_{2}+\quad+a_{n n} x_{n}=y_{n},
\end{array}\right.
$$

where the coefficient matrix $A=\left(a_{i j}\right)$ is a crisp matrix and $y_{i}$ is a fuzzy number, $1 \leq i, j \leq n$. Subsequently, lots of numerical methods were investigated for FLS (1) by a large number of authors, such as Abbasbandy, Ezzati and Jafarian [1], Allahviranloo [2], Dai, S. Wang and K. Wang [3], Dehghan and Hashemi [4], Fariborzi Araghi and Fallahzadeh [6].

In this paper, a new splitting iterative method is presented for FLS (1), compared with Jacobi method.

\section{Fuzzy linear system (FLS)}

A fuzzy number is defined as $(\underline{u}(r), \bar{u}(r)), 0 \leq r \leq 1$ which satisfies [7],

- $\underline{u}(r)$ is a bounded left continuous nondecreasing function over $[0,1]$,

- $\bar{u}(r)$ is a bounded left continuous nonincreasing function over $[0,1]$,

- $\underline{u}(r) \leq \bar{u}(r), 0 \leq r \leq 1$.

The arithmetic operations are as follows for arbitrary fuzzy numbers $x=(\underline{x}(r), \bar{x}(r))$, $y=(\underline{y}(r), \bar{y}(r)), 0 \leq r \leq 1$ and real number $k$,

(1) $x=y$ if and only if $\underline{x}(r)=\underline{y}(r), \bar{x}(r)=\bar{y}(r)$,

(2) $x+y=(\underline{x}(r)+\underline{y}(r), \bar{x}(r)+\bar{y}(r))$, and

(3) $k x= \begin{cases}(k \underline{x}(r), k \bar{x}(r)), & k \geq 0, \\ (k \bar{x}(r), k \underline{x}(r)), & k<0 .\end{cases}$

Definition 1 [7]. A fuzzy number vector $X=\left(x_{1}, x_{2}, \quad, x_{n}\right)^{\mathrm{T}}$ given by

$$
x_{i}=\left(\underline{x}_{i}(r), \bar{x}_{i}(r)\right), 1 \leq i \leq n, 0 \leq r \leq 1,
$$

is called a solution of the fuzzy linear system (1) if 


$$
\left\{\begin{array}{l}
\sum_{j=1}^{n} a_{i j} x_{j}=\sum_{j=1}^{n} \frac{a_{i j} x_{j}}{\overline{\sum_{j=1}^{n} a_{i j} x_{j}}}=\sum_{j=1}^{n} \overline{a_{i j} x_{j}}=\bar{y}_{i} .
\end{array}\right.
$$

From (2), FLS (1) can be extended to a $2 n \times 2 n$ crisp linear system

$$
S X=Y \text {, }
$$

where $S=\left(s_{k l}\right)$, and $s_{k l}$ are determined as follows

$$
\begin{aligned}
& a_{i j} \geq 0 \Rightarrow s_{i j}=a_{i j}, s_{n+i, n+j}=a_{i j}, \quad 1 \leq i, j \leq n \\
& a_{i j}<0 \Rightarrow s_{i, n+j}=a_{i j}, s_{n+i, j}=a_{i j},
\end{aligned}
$$

and any $s_{k l}$ not determined by the above items is zero, $1 \leq k, l \leq 2 n$, thus $S$ has the structure

$$
\left[\begin{array}{ll}
S_{1} & S_{2} \\
S_{2} & S_{1}
\end{array}\right]
$$

and $A=S_{1}+S_{2}$.

The following theorem guarantees FLS (1) has a unique solution.

Theorem 1 [7]. The matrix $S$ is nonsingular if and only if the matrices $A=S_{1}+S_{2}$ and $S_{1}-S_{2}$ are both nonsingular.

By Theorem 2 of [7], matrix $S^{-1}$ has the same structure as $S$, i.e.,

$$
S^{-1}=\left[\begin{array}{cc}
T_{1} & T_{2} \\
T_{2} & T_{1}
\end{array}\right],
$$

where $T_{1}$ and $T_{2}$ have the same size with $S_{1}$ and $S_{2}$.

With Theorem 1, the solution of (1) is thus unique but may still not be an appropriate fuzzy vector. Thus, the following definition is needed.

Definition 2. Let $X=\left\{\left(\underline{x}_{i}(r), \bar{x}_{i}(r)\right), 1 \leq i \leq n\right\}$ denote the unique solution of (1) from (3). If $\left(\underline{x}_{i}(r), \bar{x}_{i}(r)\right), 1 \leq i \leq n$ are all fuzzy numbers then $X$ is called a strong solution; otherwise, $X$ is called a weak solution.

\section{New iterative method for FLS}

According to $\mathrm{Du}$, Zheng andWang's new iterative methods for linear systems [5], the following iterative scheme can be established for fuzzy linear system (1), i.e., (3),

$$
X^{(k+1)}=H X^{(k)}+D^{-1} Y, \quad k=0,1,2, \quad,
$$

where $H=D^{-1} E$ with splitting

where

$$
S=D-E
$$

$$
D=\left[\begin{array}{ll}
D_{1} & \\
& D_{1}
\end{array}\right], D_{1}=\left[\begin{array}{llll}
s_{11} & & & \\
& S_{22} & & \\
& & & \\
S_{l 1} & S_{l 2} & s_{l l} & S_{\mathrm{ln}} \\
& & & \\
& & & s_{n n}
\end{array}\right],
$$

here $1 \leq l \leq n$ and all other entries are zero, and $E=-(S-D)$.

For the new iterative method (5), the following convergence theorem holds. 
Theorem 2. If $A=\left(a_{i j}\right)_{n \times n}$ satisfies $\sum_{j=1, j \neq i}^{n}\left|a_{i j}\right| \leq a_{i i}$, i.e., $A$ is diagonally dominant, then the iterative method (1) converges.

Proof. Because $S$ is of the form (4), the system (3) can be changed into two $n \times n$ systems as follows

$$
\left\{\begin{array}{l}
S_{1} \underline{X}+S_{2} \bar{X}=\underline{Y}, \\
S_{2} \underline{X}+S_{1} \bar{X}=\bar{Y}
\end{array}\right.
$$

As $A$ satisfies $\sum_{j=1, j \neq i}^{n}\left|a_{i j}\right| \leq a_{i i}, S_{1}$ satisfies $\sum_{j=1, j \neq i}^{n}\left|s_{i j}\right| \leq s_{i i}$, which meets the conditions of the convergence Theorem 3.2 of [5], thus, the method (5) converges for each $n \times n$ system of (6).

\section{Numerical examples}

In the numerical experiments, the initial guess is zero and the stopping criterion is

$$
\frac{\left\|R^{(k)}\right\|_{2}}{\left\|R^{(0)}\right\|_{2}}<10^{-6},
$$

where $R^{(k)}$ is the residual vector after $k$ iterations. In the tables, $x_{a}$ and $x_{b}$ mean that $S X=Y$ is solved as two numeric systems

not one symbolic system

$$
S\left[\begin{array}{c}
x_{a 1} \\
x_{a 2} \\
x_{a, 2 n}
\end{array}\right]=\left[\begin{array}{c}
y_{a 1} \\
y_{a 2} \\
y_{a, 2 n}
\end{array}\right] \text { and } S\left[\begin{array}{c}
x_{b 1} \\
x_{b 2} \\
x_{b, 2 n}
\end{array}\right]=\left[\begin{array}{c}
y_{b 1} \\
y_{b 2} \\
y_{b, 2 n}
\end{array}\right],
$$

$$
S\left[\begin{array}{c}
x_{a 1}+x_{b 1} r \\
x_{a 2}+x_{b 2} r \\
x_{a, 2 n}+x_{b, 2 n} r
\end{array}\right]=\left[\begin{array}{c}
y_{a 1}+y_{b 1} r \\
y_{a 2}+y_{b 2} r \\
y_{a, 2 n}+y_{b, 2 n} r
\end{array}\right]
$$

in the actual calculations. The new method (5) is compared with Jacobi method.

Example 1. Consider $n \times n$ FLS $A x=y$ with

$$
A=\left(a_{i j}\right)_{n \times n}=\left\{\begin{array}{ll}
a_{i j}=1, & i=j, \\
a_{i j}=1, & i=n, j=1, \\
a_{i j}=0, & i=n, j=2, \\
a_{i j}=-\frac{1}{n}, & \text { other } i \neq j,
\end{array}, n-1, \quad 1 \leq i, j \leq n, \quad y=\left[\begin{array}{l}
(1+r, 3-r) \\
(1+r, 3-r) \\
(1+r, 3-r)
\end{array}\right] .\right.
$$

It is seen that $A$ is a diagonally dominant matrix with positive diagonal entries. Thus, the extended system $S X=Y$ can be solved by the new method (5). The numerical results are list in Table 1 .

Table 1. Iterations (IT), CPU time (t) and relative error (ERR) for Example 1

\begin{tabular}{c|ccc|ccc|ccc|ccc}
\hline \multirow{3}{*}{$n$} & \multicolumn{7}{|c|}{ Jacobi } & \multicolumn{5}{c}{ New method } \\
\cline { 2 - 15 } & \multicolumn{4}{|c|}{$x_{a}$} & & \multicolumn{3}{|c|}{$x_{b}$} & \multicolumn{3}{c}{$x_{a}$} & \multicolumn{3}{c}{$x_{b}$} \\
\cline { 2 - 15 } & IT & $t$ & ERR & IT & $t$ & ERR & IT & $t$ & ERR & IT & $t$ & ERR \\
\hline 100 & 1308 & 0.2 & $1.0 \mathrm{e}-6$ & 1389 & 0.1 & $1.0 \mathrm{e}-6$ & 454 & 0.0 & $9.9 \mathrm{e}-7$ & 454 & 0.0 & $9.9 \mathrm{e}-7$ \\
200 & 2609 & 0.8 & $1.0 \mathrm{e}-6$ & 2771 & 0.6 & $1.0 \mathrm{e}-6$ & 915 & 0.2 & $9.9 \mathrm{e}-7$ & 915 & 0.2 & $9.9 \mathrm{e}-7$ \\
300 & 3910 & 2.5 & $1.0 \mathrm{e}-6$ & 4152 & 2.8 & $1.0 \mathrm{e}-6$ & 1375 & 0.9 & $1.0 \mathrm{e}-6$ & 1375 & 0.9 & $1.0 \mathrm{e}-6$ \\
\hline
\end{tabular}

Example 2. Consider $n \times n$ FLS $A x=y$ with 


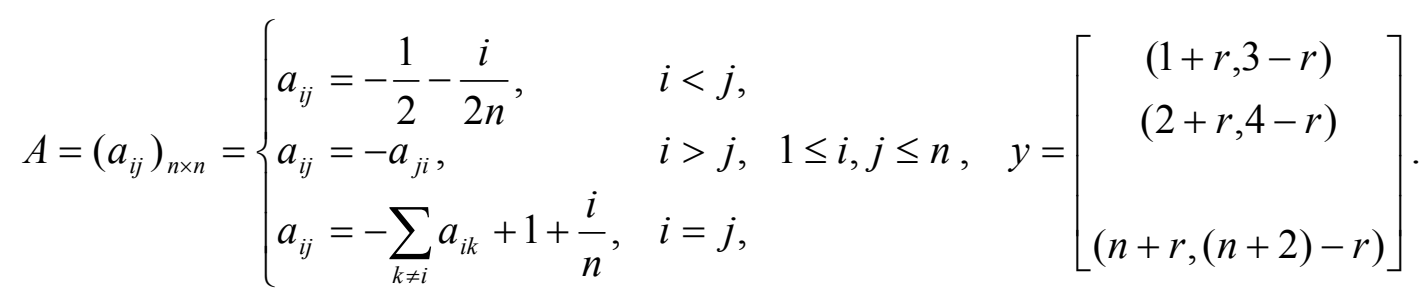

Also, $A$ is a diagonally dominant matrix with positive diagonal entries. Thus, the extended system $S X=Y$ can be solved by the new method (5). The numerical results are list in Table 2.

Table 2. Iterations (IT), CPU time (t) and relative error (ERR) for Example 2

\begin{tabular}{|c|c|c|c|c|c|c|c|c|c|c|c|c|}
\hline \multirow{3}{*}{$n$} & \multicolumn{6}{|c|}{ Jacobi } & \multicolumn{6}{|c|}{ New method } \\
\hline & \multicolumn{3}{|c|}{$x_{a}$} & \multicolumn{3}{|c|}{$x_{b}$} & \multicolumn{3}{|c|}{$x_{a}$} & \multicolumn{3}{|c|}{$x_{b}$} \\
\hline & IT & $t$ & ERR & IT & $t$ & ERR & IT & $t$ & ERR & IT & $t$ & ERR \\
\hline 100 & 1273 & 0.2 & $1.0 \mathrm{e}-6$ & 1709 & 0.1 & $1.0 \mathrm{e}-6$ & 70 & 0.0 & $5.7 e-7$ & 23 & 0.0 & $5.7 \mathrm{e}-7$ \\
\hline 200 & 2500 & 0.7 & $1.0 \mathrm{e}-6$ & 3511 & 0.8 & $1.0 \mathrm{e}-6$ & 91 & 0.0 & $7.6 e-7$ & 25 & 0.0 & $8.7 \mathrm{e}-7$ \\
\hline 300 & 3711 & 2.4 & $1.0 \mathrm{e}-6$ & 5350 & 3.3 & $1.0 \mathrm{e}-6$ & 113 & 0.1 & $9.3 e-7$ & 27 & 0.0 & $5.0 \mathrm{e}-7$ \\
\hline
\end{tabular}

Tables 1 and 2 give the number of iterations, CPU time and precision. The results show that the new method is much better than Jacobi method.

\section{Conclusions}

In this paper, a new iterative method is discussed for solving the fuzzy linear systems proposed by Friedman et al [7]. The convergence theorem shows that the method converges for diagonally dominant systems with positive diagonal entries. Numerical examples demonstrate that the new method outperforms Jacobi method.

\section{Acknowledgements}

This work was financially supported by National Natural Science Foundation of China (11301330) and the grants of "The First-class Discipline of Universities in Shanghai" and "Gaoyuan Discipline of Shanghai".

\section{References}

[1] S. Abbasbandy, R. Ezzati and A. Jafarian: Appl. Math. Comput., Vol. 172 (2006), p. 633

[2] T. Allahviranloo: Appl. Math. Comput., Vol. 155 (2004), p. 493

[3] S. Dai, S. Wang and K. Wang: Comput. Math. Model., Vol. 26 (2015), p. 577

[4] M. Dehghan and B. Hashemi: Appl. Math. Comput., Vol. 175 (2006), p. 645

[5] J. Du, B. Zheng and L. Wang: J. Appl. Anal. Comput., Vol. 1 (2011), p. 351

[6] M.A. Fariborzi Araghi and A. Fallahzadeh: Soft Comput., Vol. 17 (2013), p. 159

[7] M. Friedman, M. Ming and A. Kandel: Fuzzy Sets and Systems, Vol. 96 (1998), p. 201 